\title{
Mobster: accurate detection of mobile element insertions in next generation sequencing data
}

\author{
Djie Tjwan Thung ${ }^{1}$, Joep de Ligt ${ }^{1,4}$, Lisenka EM Vissers ${ }^{1}$, Marloes Steehouwer ${ }^{1}$, Mark Kroon ${ }^{2}$, Petra de Vries ${ }^{1}$, \\ Eline P Slagboom², Kai Ye ${ }^{3}$, Joris A Veltman ${ }^{1,5}$ and Jayne Y Hehir-Kwa ${ }^{{ }^{*}}$
}

\begin{abstract}
Mobile elements are major drivers in changing genomic architecture and can cause disease. The detection of mobile elements is hindered due to the low mappability of their highly repetitive sequences. We have developed an algorithm, called Mobster, to detect non-reference mobile element insertions in next generation sequencing data from both whole genome and whole exome studies. Mobster uses discordant read pairs and clipped reads in combination with consensus sequences of known active mobile elements. Mobster has a low false discovery rate and high recall rate for both L1 and Alu elements. Mobster is available at http://sourceforge.net/projects/mobster.
\end{abstract}

\section{Background}

Mobile elements (MEs) or transposable elements are DNA sequences that can be autonomously copied or moved through the genome, yet their highly repetitive sequence structure makes them difficult to detect. In addition to being a major evolutionary driver in changing the genomic architecture, MEs have also directly resulted in pathogenic variation in a number of human diseases by inserting into functionally important regions and disrupting gene function [1,2]. MEs can be classified into two different classes depending on their mode of transposition. Class I retrotransposons travel through an RNA intermediate by copy and paste, while class II DNA transposons have a DNA intermediate and generally move by cut and paste. Together these elements make up the majority of the human genome, with estimates in the range of $45 \%$ to $69 \%$ of the human genome sequence belonging to one of these transposons classes [3,4].

Currently only a few MEs remain active or 'hot' in the human genome, all of which belong to the retrotransposon class and include the autonomous L1 family (6 kb, 500,000 copies), the non-autonomous Alu (300 bp, 1,000,000 copies) and SVA ( $2 \mathrm{~kb}, 3,000$ copies) families [5-8]. These ME families continue to change the genomic architecture by inserting into new regions in the DNA, transducing DNA, shuffling exons, and creating processed pseudogenes. Even

\footnotetext{
* Correspondence: Jayne.Hehir-Kwa@radboudumc.nl

'Department of Human Genetics, RadboudUMC, P.O. Box 9101, 6500, HB,

Nijmegen, the Netherlands

Full list of author information is available at the end of the article
}

ancient and inactive relics of transposition have a major contribution to genomic variation as their sequence homology can lead to unequal crossing over, resulting into deletions or duplications of DNA between two ME copies [9].

Transposition of MEs often occurs in the germline or during early embryogenesis. The first disease-causing ME insertion (MEI) in humans was found in exon 14 of the FVIII gene in two patients with hemophilia A [10]. Since then over 90 disease-producing MEIs have been found, consisting of 60 insertions of Alu elements, 25 insertions of L1s, and seven insertions of SVA [8]. Furthermore MEs are known to play a role in cancer development and tumor-specific MEI events have been found in several studies [11-13].

To identify polymorphic MEIs (pMEIs), both targeted and next-generation sequencing (NGS) analysis have been developed. Previous attempts to computationally detect pMEIs in human NGS data generally use discordant read pairs or clipped reads to identify pMEIs. Hormozdiari et al. modified VariationHunter to characterize polymorphic Alu insertions [14], while Ewing and Kazazian developed a pipeline for detecting polymorphic L1 insertions [15]. Tea [13] and RetroSeq [16] can use clipped reads in addition to discordant pairs to fine tune the breakpoints of the MEI event. Finally an unpublished pipeline from Stewart et al. can use a split-read method to detect pMEIs in longer 454 reads in addition to a paired-end approach for paired-end Illumina data [17]. 
We present a novel method, named Mobster, which is able to detect active non-reference MEIs with high accuracy in both WGS and WES data. Furthermore our method is not limited to a specific family of MEI events but is able to detect all families of active MEI events. Our method outperforms existing tools on a public human dataset, as well as simulation data with varying coverage. We then applied Mobster to a variety of NGS data types which include a paired-end WGS dataset, a paired-end WES dataset, and a single-end WES dataset, and performed PCR validation experiments.

\section{Materials and methods}

\section{The mobster method}

Mobster uses a combination of discordant read pairs and clipped reads in binary alignment (BAM) files to search for candidate active non-reference MEI events (Figure 1). Read pairs are considered to be discordant when: (1) the orientation of the mapped reads differs from the expected orientation; (2) the distance between mapped reads differs significantly from the median insert size; (3) reads are mapped to different chromosomes; or (4) one read is mapped, while the other read is unmapped. Discordant pairs which have at least one uniquely mapped read are used to anchor the possible insertion event. The mates of the anchoring reads are then mapped to a custom but configurable library of known active ME consensus sequences (mobilome, Additional file 1: Table S2). When a discordant pair contains two uniquely mapped reads, both reads are mapped to the mobilome, subsequently excluding reads which both map to a ME. If the BAM file contains clipped reads, the clipped sequences of uniquely mapped reads are also mapped against the mobilome and investigated for a polyA or polyT stretch. Anchoring reads are tagged as either unmapped or according to the mapping of their mate or clipped sequence to a ME family in the mobilome (Alu, L1, SVA, or HERV-K). If the data have been generated using paired-end libraries, first the discordant pair anchors supportive for the same ME family are clustered together: anchors which are within a user-specified neighborhood distance and map to the same reference strand are clustered together. Subsequently

A
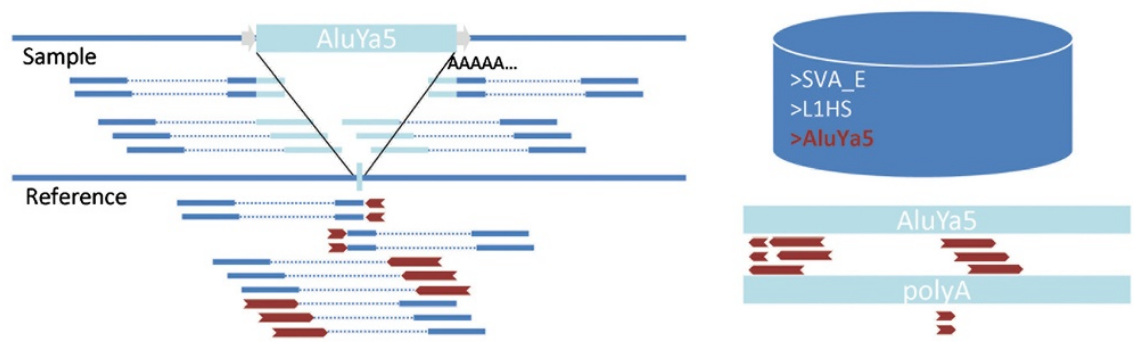

B

(1)

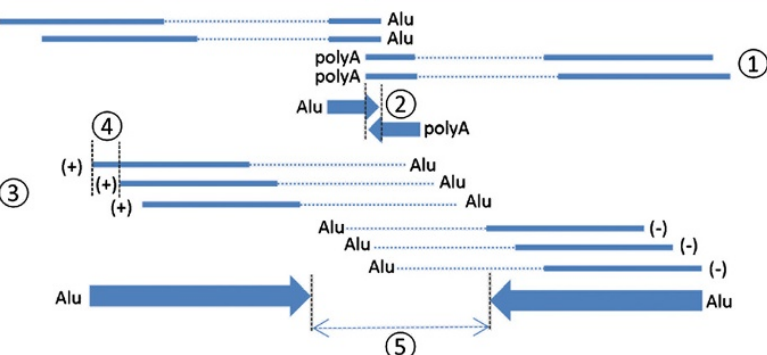

Figure 1 Overview of the Mobster algorithm. (A) In the first phase discordant ends (long red arrows) and clipped ends (short red arrows) are extracted from the BAM file when, respectively, the mate or the unclipped end is mapped uniquely to the reference. Subsequently these reads are mapped to the mobilome and investigated for having a polyA/T tail. (B) After mapping, all mates and unclipped sequences (anchors) belonging to unambiguously mapped Alu, L1, SVA, or HERV-K reads are identified. Anchors of clipped reads are clustered separately from anchors of discordant reads. (1) For clipped clusters, anchors should be: (i) supportive of the same ME family or same polyA/T stretch; (ii) clipped on the same side; and (iii) clipped within a few bp of each other. (2) The 5' clipped cluster (arrow pointing to right), consisting of right-clipped reads, and $3^{\prime}$ clipped cluster (arrow pointing to left), consisting of left-clipped reads, are indicative of the same MEl event when: (i) they support the same ME family or one of the clusters supports a ME family and the other cluster supports a polyA/T tail; and (ii) they overlap by a maximum of $50 \mathrm{bp}$ (allowing for TSDs) or are separated by a maximum of $20 \mathrm{bp}$ (allowing for target site deletions). $(3,4)$ Discordant pair anchors, are clustered when: (i) they map to the same strand; (ii) are supporting the same ME family; and (iii) have start positions, which are within a specified neighborhood distance (4). (5) Forward strand anchors form 5' discordant clusters; reverse strand anchors form $3^{\prime}$ discordant clusters. Discordant clusters from the $5^{\prime}$ and $3^{\prime}$ are indicative of the same MEl event when they overlap by maximal $50 \mathrm{bp}$ or are within a user-defined window size. When possible, clipped clusters are merged with discordant clusters. 
forward-strand and reverse-strand clusters indicative of the same MEI event are joined together if possible, creating both double clusters (support on $5^{\prime}$ end and 3 ' end of putative MEI event) and single clusters. In addition to discordant pairs, clipped reads indicative for MEI are also clustered based on whether they are clipped on the right side ( $5^{\prime}$ clipped cluster) or left side ( $3^{\prime}$ clipped cluster) and map to the same ME family or to the same homopolymer (A or T). 5' Clipped clusters and 3' clipped clusters are joined together when both clusters map to the same ME family or when one of the clusters is indicative for a polyA or polyT stretch and the other is indicative for a specific ME family. Finally the clusters containing anchors from discordant pairs and clipped clusters are joined together. Breakpoints are estimated from the inner borders of the 5 ' clipped and 3' clipped cluster or when not available from the inner borders of discordant pair clusters. For single cluster predictions with no available clipped reads, breakpoints and prediction window borders are estimated from the insert size distribution, calculated by Picard's CollectInsertSizeMetrics [18], and the length of the cluster itself. To avoid predicting MEIs already present in the reference, all predictions are filtered with a prediction window within $90 \mathrm{bp}$ of an annotated MEI of the same ME family as the predicted MEI. In single-end libraries only clipped reads are used to predict MEI events.

Default filtering of predictions is based on events with less than five supporting reads. Supporting reads are defined, in the case of split reads, as reads that partially map uniquely to the reference genome, and partially to a ME or polyA tail. In the case of discordant reads, they refer to reads in which one end of the read pair uniquely maps to the reference genome and the second end maps to a mobile element. Furthermore data presented here are generated using Mobster with 2GB memory using 8 2.67GHz CPUs, with the exception of the CEU trio for which $8 \mathrm{~GB}$ memory was used a comparison between the resource usage of Mobster and additional MEI identification tools is presented in (Additional file 1: Table S3).

\section{The mobilome and annotations}

A mobilome reference database was made by selecting a subset of RepBase consensus sequences of 54 MEs thought to be still active in humans [5]. Sequences were extracted from RepBase version 17.3 [19] and include sequences from the Alu, L1, SVA, and HERV-K families (see Additional file 1: Table S2).

Mobster's predictions were annotated with previously reported pMEIs found in dbRIP [16] and with novel events reported in healthy tissue and predicted in silico by Stewart et al. [17], Hormozdiari et al. [14], and Lee et al. [13] using a 50 bp window based on the prediction boundaries of Mobster's calls. When necessary, hg18 coordinates of previously predicted pMEIs were converted to hg19 using the UCSC liftOver tool. To determine whether predictions coincide with a known gene and gene components the refSeq genes for hg19 were downloaded from UCSC. Predicted MEIs were annotated with refSeq genes using ANNOVAR [20], reporting one gene component per prediction. If a prediction overlapped with multiple gene components, for example a coding exon and an intron, priority was given to components in the following order: (1) exons, splice sites; (2) ncRNA; (3) UTRs; and (4) introns.

\section{Insertion and orientation bias}

A UCSC custom track containing only introns was created from the refSeq genes, including alternative transcripts. Using an in-house strand-aware python script all first and last introns were extracted from the resulting BED file. With bedtools 2.15 [21] sort and merge three sets of nonredundant regions were created: all introns, first introns, and last introns. To avoid bias from genes with one intron populating only one of the subsets, these introns were included in all three sets. The hypothesized probability of a MEI inserting into the first intron of a gene was based upon the genomic size of all non-redundant first introns divided by the total size of all non-redundant introns. This hypothesized probability was tested against the observed fraction of intronic MEIs in first introns using binomial testing. Orientation of MEI events is determined by the detection of a poly A tail: $3^{\prime}$ detected poly A is considered the plus $(+)$ strand, while $5^{\prime}$ poly $\mathrm{A}$ is considered minus (-) strand. The orientations of human genes were extracted from refSeq genes from UCSC.

DNA sequence insertion bias around the breakpoints was assessed for MEI events having target site duplications and consistent clipping positions of clipped clusters. For plus strand and negative strand insertions a $13 \mathrm{bp}$ reference subsequence was extracted around the clipping position of the $3^{\prime}$ clipped cluster and the $5^{\prime}$ clipped cluster, respectively. Sequence logos were created for plus strand insertions and minus strand insertions separately with WebLogo 3.3 [22].

\section{Benchmarking with CEU trio}

The performance of Mobster was benchmarked against predictions made with four different algorithms (RetroSeq [16], Tea [13], Tangram [23] and, alu-detect [24]) using a publically available data. This dataset consists of high coverage ( $>60 \mathrm{X})$ CEU trio (NA12878 female child, NA12891 father, NA12892 mother) available through [25]. Published PCR MEI validations were used to compare the recall of mobster with other tools [17]. The MEI call sets for RetroSeq [16], Tea [13] and Tangram were downloaded from [26], while alu-detect version 1.3 was run using default settings on NA12878. Alu-detect failed to complete analysis on NA12891 and NA12892. 
Mobster was run requiring a minimum of five supporting reads, a neighborhood distance of $200 \mathrm{bp}$, and a maximum distance between single discordant clusters of $600 \mathrm{bp}$ in order for them to be joined. Clipped sequences needed to be at least $35 \mathrm{bp}$ long with a minimum average base quality of 20, while the read may not be clipped at the other end for more than $7 \mathrm{bp}$, after which only double cluster predictions were considered. De novo calls were defined as calls in the child not overlapping with a call in the parents within a $50 \mathrm{bp}$ merging window for Mobster and a $200 \mathrm{bp}$ for the other algorithms. Similarly the overlap between Mobster and the PCR validated events was based on a 50 bp window, whilst 200 bp was allowed for the other algorithms and matching ME family predicted. Different merging windows were used for a fairer comparison between the tools: on average the prediction windows made by Mobster were larger than those made by the other tools. By increasing the merging window for these tools, the recall of the PCR validated events was increased and false de novo calls were reduced. Furthermore the whole exome data for this CEU trio were downloaded from the same ftp site for comparison between detection of MEI events with whole genome sequencing data. The whole exome capture regions were obtained from [27].

\section{WGS and WES experimental datasets}

Whole genome paired-end sequencing data were obtained by sequencing the DNA from healthy Dutch monozygotic twins to an average depth of 40X (EGAS00001000877), using the Illumina HiSeq platform (Illumina, Inc., San Diego, CA, USA) and paired-end libraries (Table 1) and aligned to the human reference (hg19) using BWA version 0.5.7-5 [28] with default settings. Mobster was then run, requiring a minimum of five supporting reads, a neighborhood distance of $200 \mathrm{bp}$, and a maximum distance between single discordant clusters of $600 \mathrm{bp}$ in order for them to be joined. Clipped sequences needed to be at least $35 \mathrm{bp}$ long with a minimum average base quality of 20 , while the read may not be clipped at the other end for more than $7 \mathrm{bp}$. Putative MEI reads were mapped against the mobilome using MOSAIK v2.1.33 [29], allowing up to $10 \%$ mismatches. Similarly whole exome paired-end sequencing data were obtained for a trio and a fourth

Table 1 Characteristics of the three different experimental datasets used to test MEl identification with Mobster

\begin{tabular}{llll}
\hline & $\begin{array}{l}\text { Whole genome } \\
\text { paired-end }\end{array}$ & $\begin{array}{l}\text { Whole exome } \\
\text { paired-end }\end{array}$ & $\begin{array}{l}\text { Whole exome } \\
\text { single-end }\end{array}$ \\
\hline $\begin{array}{l}\text { Number samples } \\
\text { Average depth of }\end{array}$ & 2 & 4 & 300 \\
coverage & $95 \mathrm{X}$ & $40 \mathrm{X}$ \\
$\begin{array}{l}\text { Read length } \\
\text { Sequencing platform }\end{array}$ & Illumina & Illumina & SOLiD \\
\hline
\end{tabular}

unrelated individual sequenced to high depth (95X) using a paired-end library with the Illumina HiSeq platform (EGAS00001000852) and aligned by BWA 0.5.9 [28] using default settings and the same settings for Mobster and MOSAIK as for the WGS data. Finally 101 trios of an intellectual disability patient cohort were sequenced with the SOLiD 4 system, generating 50 bp single-end reads with an average depth of 40X [30]. The short reads were mapped in colorspace to the human genome (hg19) reference using Life Technologies proprietary software, BioScope version 1.2 , which uses an iterative alignment strategy. This iterative alignment strategy, clips bases at either the $5^{\prime}$ end or $3^{\prime}$ end of the read on each iteration, to map the read if global alignment of the read against the reference was not successful. A maximum of $15 \mathrm{bp}$ hard-clipping was allowed for the $5^{\prime}$ end, while a maximum of $25 \mathrm{bp}$ hard-clipping was allowed for the 3' end.

\section{Validation experiments}

Eleven random MEI events were selected for validation from the WGS dataset representing the following categories: (1) novel MEI calls predicted by Mobster and a second algorithm developed at the Leiden University Medical Center (LUMC) (unpublished); (2) novel MEI calls predicted by Mobster alone; (3) MEI calls predicted by previous in silico tools or existent in dbRIP and predicted by both Mobster and the LUMC algorithm; (4) MEI calls predicted by Mobster and existent in dbRIP or predicted by previous in silico tools. Similarly 10 random MEI events from the WES paired-end data were selected for validation. PCR primers were designed with a minimum of $50 \mathrm{bp}$ upand downstream the estimated insertion breakpoint.

Prior to the validation of MEI events, all primers and PCR conditions were tested and optimized using control DNA ensuring correct amplification. For MEI events with a predicted insertion size smaller than $1 \mathrm{~kb}$ and insertions estimated to be larger than $1 \mathrm{~kb}$, different amplifications kits were used, being REDtaq readymix (Sigma-aldrich) and RangerDNA Polymerase (Bioline), respectively (PCR conditions available upon request). Subsequently, PCR amplicons were checked on agarose gels. Amplicons consistent with the presence of a hetero- or homozygous insertion were analyzed by capillary (Sanger) sequencing using routine procedures. Sequences obtained were analyzed using Vector NTI. The class of ME was determined by using a Blat search of the inserted sequences in the UCSC browser.

\section{Results}

MEs play an active role in modifying genomic structure. However, due to their highly repetitive nature they are difficult to detect using short-read NGS technologies. We have developed a novel method (Mobster) to detect active non-reference MEI events in both whole exome 
and whole genome, based on single-end and paired-end sequencing data. The accuracy of Mobster was tested on both simulation (see Additional file 1: Results) and experimental human NGS data.

\section{Accuracy based on simulation data}

The accuracy of Mobster was first tested on WGS simulation data with depths ranging from 10X to 160X. These results show a very high sensitivity (99.1\%) with as little as 10X coverage for homozygous MEI events reporting no false positives (Additional file 1: Figures S1-2). At the highest coverage (160X), sensitivity is marginally improved to $99.9 \%$, with one false positive. Paired-end WES simulation show a markedly lower sensitivity, ranging from $52.7 \%$ to $85.4 \%$ at $10 \mathrm{X}$ to $160 \mathrm{X}$, respectively. This reflects the difficulty of identifying MEI events in small exonic capture regions, likely influenced by the simulation capturing on target but not near target sites. Overall the positive predictive value of the algorithm is good, ranging from $98.5 \%$ to $99.9 \%$ at the highest and lowest coverage, respectively.

\section{Accuracy based on CEU trio}

The accuracy of Mobster was first tested on a WGS, high coverage, public dataset consisting of the CEU trio (NA12878, NA12891, NA12892) and compared against previous results. Tea, Mobster and RetroSeq detected around 1,200 to 1,250 Alu and L1 events in NA12878, while Tangram and alu-detect predicted between 1,550 and 1,620 events (Additional file 1: Table S4 and Additional files 2,3 , and 4). The trio provides a good way to benchmark the different algorithms as expected number of de novo MEI events lie between 0 and 2 . A higher amount of de novo events than expected can indicate both false positives in the child and false negatives in the parents. Mobster had the lowest percentage of de novo MEI events $(4.5 \%, \mathrm{n}=54)$ in comparison to RetroSeq [16] $(7.7 \%, \mathrm{n}=$ 97), Tangram (12.4\%, $\mathrm{n}=192)$, and Tea [13] $(14.3 \%, \mathrm{n}=$ 172) (Additional file 1: Table S4). All of the predicted events in this trio, including those which were inherited, were then compared with PCR validated events (Table 2). Of the 1,029 Alu events validated across the three individuals, Mobster detected 1,015 (98.7\%), in comparison to $98.1 \%$ by Tangram, $97.8 \%$ by RetroSeq, $95.1 \%$ by aludetect, and $91.1 \%$ by Tea. In addition Mobster detected 89 of the $99(89.8 \%)$ validated L1 events in this trio, whereas Tangram detected $85.5 \%$, RetroSeq $83.7 \%$, and Tea $80.7 \%$.

\section{Detecting MEls in paired-end WGS experimental data}

Mobster was then tested on WGS NGS 40X experimental data from monozygotic twins requiring a minimum support of five sequencing reads (see Methods). Furthermore clipped reads were required to have a length of at least 35 bp with a minimum average base quality of 20 .
Table 2 Percentage of PCR validated events recalled from CEU trio by the different algorithms

\begin{tabular}{lll}
\hline & Alu events & L1 events \\
\hline Nr PCR events & 1029 & 99 \\
Mobster & $\mathbf{9 8 . 7}$ & $\mathbf{8 9 . 8}$ \\
Tangram & 98.1 & 85.5 \\
RetroSeq & 97.8 & 83.8 \\
alu-detect & $95.1^{\mathrm{a}}$ & $\mathrm{NA}$ \\
Tea & 91.1 & 80.7 \\
\hline
\end{tabular}

${ }^{a}$ Recall calculated based on NA12878 (408 PCR validated Alu events). Values in bold depict best performing algorithm.

We discarded MEI predictions which only had supporting reads at one side of the insertion and those for which the predicted insertion coordinates were within satellite DNA. In addition predicted events occurring within 50 bp of each other were merged. Using this strategy a total of 1,179 MEIs were identified, 1,068 of which were shared between the samples, resulting in a $90.6 \%$ overlap (Additional file 1: Figure S4a).

We hypothesize the remaining 111 private variants were in fact false negatives in the other sibling. We investigated this by pooling supporting anchoring reads data from both samples and reprocessing with Mobster. Each prediction required to have at least five supporting reads from one sample. The same filtering steps were used as for the non-pooled analysis, resulting in 1,181 called MEIs with no private variants, supporting our false negative hypothesis (Additional file 1: Figure S4b). By considering all predictions from one sibling to be true positives in the non-pooled analysis, then the estimated false negative rate for Mobster ranges between $3.6 \%$ for sibling $\mathrm{C}$ and $5.9 \%$ for sibling $\mathrm{A}$.

The majority of the predicted MEIs show hallmarks of retrotransposition. In 954 events a reliable estimate could be made whether a MEI was associated with an indel at the site of integration. The vast majority of MEIs $(n=889)$ were inserted with a target site duplication (TSD), with a minority having a target site deletion $(n=57)$ or no indel $(\mathrm{n}=8)$. The median TSD size of all MEI events was $13 \mathrm{bp}$, while the median deletion size was 7 bp (Figure 2A). Furthermore in 735 out of 753 predicted MEIs supported by clipped reads at both sides of the event a polyA tail longer than 8 bp could be detected.

The majority of MEI events detected were $A l u$ in origin, followed by L1 and SVA $(85.4 \%, 11.4 \%$, and $3.2 \%$, respectively), (Figure 2B). No HERV-K insertions were identified. Using a $50 \mathrm{bp}$ merging window the vast majority (95.5\%) of predicted events overlap with either previous in silico predictions in healthy individuals or with entries in the database of retrotransposon insertion polymorphisms (dbRIP). Only 436 of the 1,181 MEIs (36.9\%) were predicted to occur within refSeq genes, 


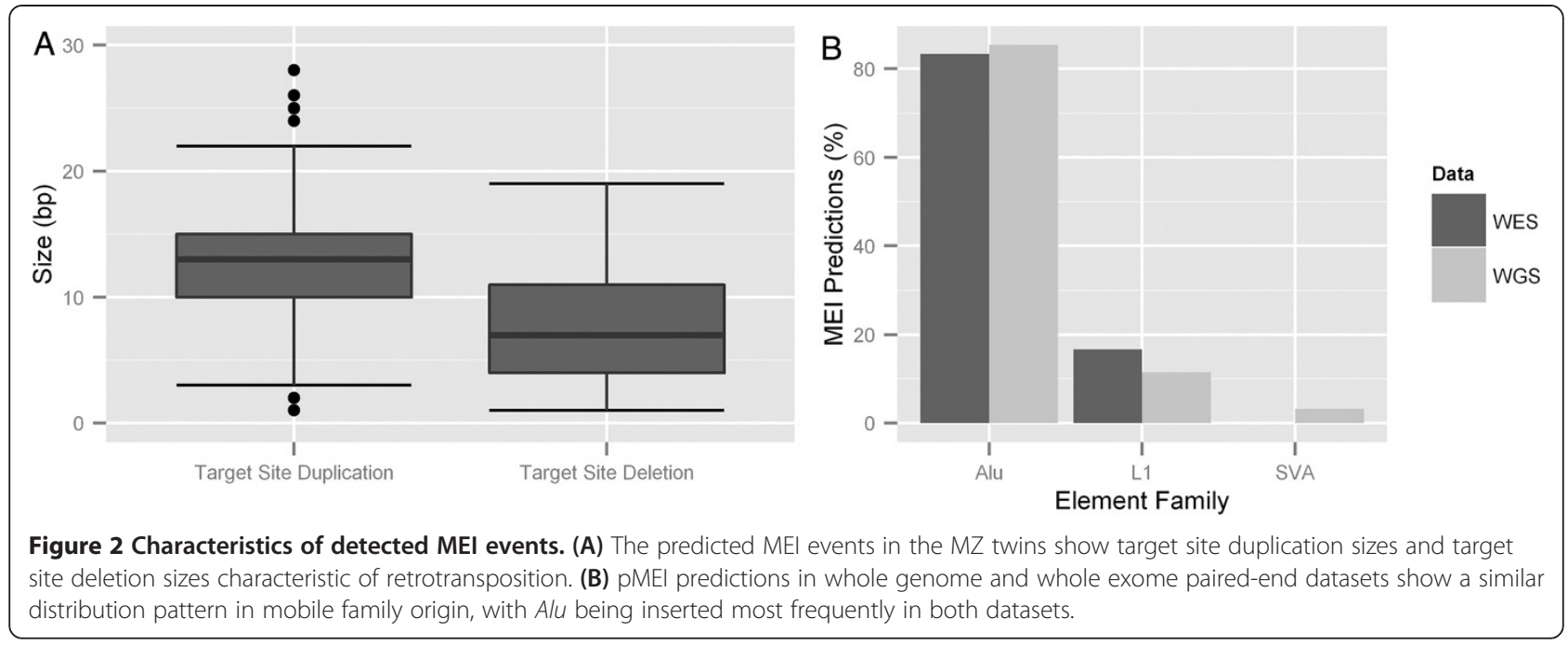

while refSeq genes makes up $42.8 \%$ of the callable genome (Table 3). This represents a significant depletion of genic MEI events $\left(P=2.1 \times 10^{-5}\right.$, one-sided binomial testing). In addition only one MEI had a potential overlap with an exon, situated near the border of a DSCAM exon. Apart from the depletion of MEIs in genic regions, the locations of the MEIs seem to be randomly distributed across the genome, with $5 \mathrm{Mb} \mathrm{MEI}$ bin counts following the Poisson distribution closely $(\lambda=2.46$, considering $6335 \mathrm{MB}$ bins in a $3.1 \mathrm{~GB}$ genome). A strong motif signal surrounding the breakpoints of MEIs with target site duplications was observed, TTTT/A[AT], with the slash indicating the breakpoint (Additional file 1: Figure S5). This suggests the majority of events are mediated by L1 endonucleases as their most common target sequence is TTTTAA [31].

Next we investigated the orientation and insertion bias of the 427 MEIs in intronic sequences. No significant insertion bias was observed, but a slight trend was observed towards depletion of $A l u$ insertions in first introns: $26.4 \%$

Table 3 Gene components affected by MEI events in healthy individuals sequenced with WGS

\begin{tabular}{ll}
\hline Genomic component & Predictions (n) \\
\hline Genic & $436(36.9 \%)$ \\
Coding gene exonic & $1(0.1 \%)$ \\
Coding gene intronic & $395(33.4 \%)$ \\
Coding gene UTR5 & 4 \\
Coding gene UTR3 & $1(0.1 \%)$ \\
Non-coding gene exonic & $3(0.3 \%)$ \\
Non-coding gene intronic & $32(2.7 \%)$ \\
Non-genic & $745(63.1 \%)$ \\
1 kb downstream TSS & $6(0.5 \%)$ \\
1 kb upstream TSS & $5(0.4 \%)$ \\
Intergenic & $734(62.2 \%)$ \\
\hline
\end{tabular}

of 427 Alus are inserted in first introns compared to an expected percentage of $29.6 \%(P=0.18$, two-sided binomial testing). While SVAs insertions $(n=12)$ tend to be enriched in last introns with an observed percentage of $25 \%$ and an expected percentage of $11.1 \%(P=0.14$, twosided binomial testing) (Additional file 1: Figure S6). The orientation of 269 intronic MEIs could reliably be detected. Intronic MEIs show a significantly higher number of insertions in the opposite orientation of the gene they were inserted into; $59.5 \%$ of insertions being in opposite sense $\left(P=2.2 \times 10^{-3}\right)$.

In total 10 of the 11 events selected for validation were successfully PCR validated (FDR $=9 \%$ ) (Table 4, Figure 3). Validated events were present in both heterozygous ( $\mathrm{n}=$ 9) and homozygous $(\mathrm{n}=1)$ states, and included insertion events representing the three main ME families (Alu, L1, and SVA). Three of the validated events were novel, previously not reported in dbRIP [16] or in in silico predictions of other studies $[13,14,17]$. In six events, Sanger sequencing of the PCR products confirmed the ME families predicted by Mobster. In the remaining five events Sanger sequences remained inconclusive.

\section{Detecting MEls in paired-end WES experimental data}

To test the performance of Mobster on WES paired-end NGS data a trio and an unrelated individual sequenced to high depth using a paired-end library were analyzed (Table 1). Mobster identified on average 22 MEI events (range, 20 to 23) per individual, with a total of 87 predictions. All predictions required to have at least five supporting unduplicated reads. Support on both the $5^{\prime}$ end and $3^{\prime}$ end side of the insertion site was not required, allowing Mobster to detect insertions near the borders of the exome capture region. After merging all predictions into a unique set of MEIs, 42 loci remained. The majority of these 42 MEIs were Alu in origin (83.3\%), 
Table 4 Validation of MEI detection in WGS and WES paired-end data

\begin{tabular}{|c|c|c|c|c|c|c|c|c|}
\hline \multicolumn{9}{|c|}{ Whole genome } \\
\hline Chr & Predicted insertion point & MEI & Gene component $^{a}$ & Gene name & Genotype & TSD & Novel $^{b}$ & Validated \\
\hline chr1 & $60,470,596$ & Alu & Intronic & Clorf87 & Heterozygous & Duplication & & Yes \\
\hline chr1 & $83,201,791$ & L1 & Intergenic & & Heterozygous & Duplication & Yes & Yes \\
\hline chr1 & $93,167,519$ & Alu & Intronic & EVI5 & Homozygous & Unknown & & Yes \\
\hline chr1 & $142,803,597$ & L1 & Intergenic & & Homozygous reference & Duplication & Yes & No \\
\hline chr3 & $103,171,382$ & Alu & Intergenic & & Heterozygous & Deletion & & Yes \\
\hline chr4 & $80,883,493$ & Alu & Intronic & ANTXR2 & Heterozygous & Duplication & Yes & Yes \\
\hline chr8 & $53,791,040$ & Alu & Intergenic & & Heterozygous & Duplication & & Yes \\
\hline chr8 & $132,672,106$ & Alu & Intergenic & & Heterozygous & Duplication & Yes & Yes \\
\hline chr10 & $130,625,059$ & L1 & Intergenic & & Heterozygous & Duplication & & Yes \\
\hline chr17 & $43,660,608$ & SVA & Intergenic & & Heterozygous & Unknown & & Yes \\
\hline chr20 & $29,638,569$ & L1 & Upstream & MLLT1OP1 & Heterozygous & Duplication & & Yes \\
\hline \multicolumn{9}{|c|}{ Whole exome } \\
\hline Chr & Predicted insertion point & MEI & Gene component ${ }^{a}$ & Gene name & Genotype & TSD & Novel $^{b}$ & Validated \\
\hline chr1 & $93,167,519$ & Alu & Intronic & EVI5 & Homozygous & Unknown & & Yes \\
\hline chr2 & $11,426,360$ & Alu & Intronic & ROCK2 & Homozygous & Unknown & & Yes \\
\hline chr3 & $50,879,159$ & Alu & Exonic & DOCK3 & Homozygous & Unknown & & Yes \\
\hline chr5 & $173,036,482$ & L1 & Exonic & BOD1 & NA & Unknown & Yes & No \\
\hline chr6 & $52,712,717$ & Alu & Intergenic & & Homozygous & Unknown & & Yes \\
\hline chr9 & $68,415,155$ & Alu & Intergenic & & NA & Unknown & & $Y_{e s}^{c}$ \\
\hline chr11 & 428,014 & Alu & Intronic & ANO9 & Homozygous & Unknown & & Yes \\
\hline chr11 & $112,084,617$ & L1 & Intronic & $\mathrm{BCO} 2$ & Heterozygous & Unknown & & Yes \\
\hline chr17 & $61,565,890$ & Alu & Intronic & $A C E$ & Heterozygous & Unknown & & Yes \\
\hline chr19 & $52,888,055$ & Alu & Exonic & ZNF880 & Homozygous & Duplication & & Yes \\
\hline
\end{tabular}

${ }^{a}$ Overlap with gene component is determined based on Mobster's predicted insertion window.

${ }^{\mathrm{b}}$ Not overlapping dbRIP or in silico MEI predictions $[13,14,17]$ within a 50 bp window.

c 454 validation by Stewart et al.

On average 1,181 MEI events were detected per WGS sample of which $4.5 \%$ were novel. Ten of the 11 randomly select MEI events could be validated. MEI detection in WES produced on average 42 events per exome of which $4.8 \%$ were novel. Nine of the 10 randomly selected MEl events from the WES predictions could be validated.

$\mathrm{TSD}=$ target site duplication

with the remaining events reported to be L1 (16.7\%). The predicted insertion windows of four MEIs (9.5\%) overlapped with exons from ANO5, ZNF880, DOCK3, and $B O D 1$. In addition the vast majority of events (95.2\%) had previously been reported in either dbRIP or literature $[13,14,17]$.

We focused on the parent-child trio to determine the inheritance characteristics of the MEIs. Out of the 21 insertion events in the child, 17 could be identified in at least one parent, leaving four potential de novo events. However these MEIs were all called near the borders of captured exonic regions, where coverage is low. Hence we hypothesized these events may have been missed in the parents by using a cutoff of five supporting reads. To test this hypothesis the sequence data from all individuals in the trio were pooled and MEI events identified, subsequent analysis of the child confirmed that these four events were false negatives in one of the parents and no de novo MEI events could be detected (Additional file 1: Figure S7).

A subset of 10 random events from the 42 predictions was chosen for validation. By PCR, gel electrophoresis, Sanger sequencing, or 454 sequencing nine of the 10 events were validated (Table 4, example of validated event in Figure 3A to C). These events included mostly one-sided predictions of insertions from Alu and L1 into intergenic, intronic, and exonic regions. For eight events Sanger sequencing of the PCR products of the insertion allele was concordant with the predicted MEI. In addition a ninth event, an Alu insertion into an intergenic region on chromosome 9 , was previously validated using 454 sequencing [17]. The remaining 10th prediction was predicted in a parent and a child to be located in exon 3 of BOD1. However in both the parent and the child clipped reads were found, with ends matching perfectly 
A

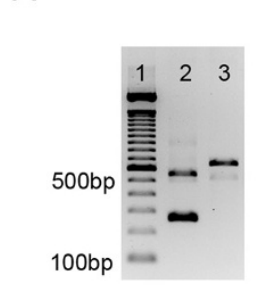

D

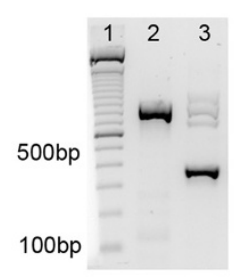

B

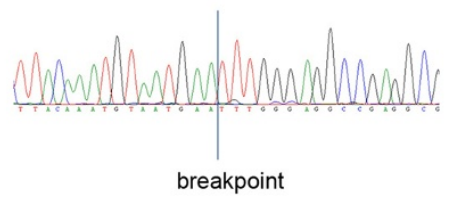

$\mathrm{E}$

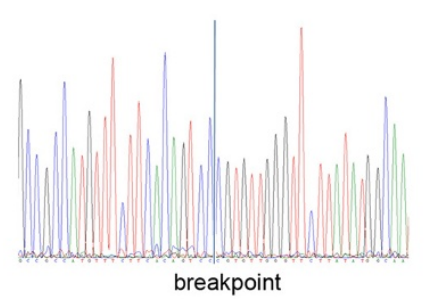

C

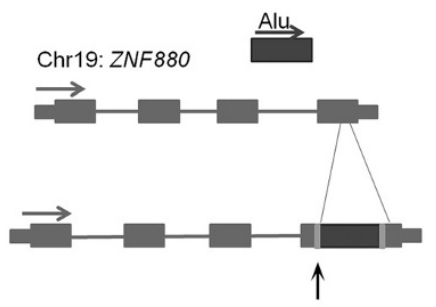

$\mathrm{F}$

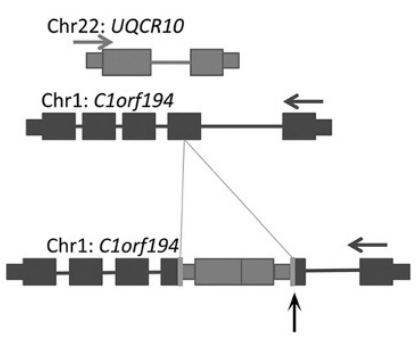

Figure 3 Validation of MEl events detected. (A) Validation of Alu events, bp in brackets correspond to the expected PCR product size of the wild-type allele. 1: 100 bp marker, 2: WES event10 homozygous MEl insertion (178 bp). (B) Sanger trace of first breakpoint. (C) Schema representing exonic Alu insertion in ZNF880. (D) Single-end exome sequencing reveals a novel processed pseudogene (UQCR10) insertion into the exon of C10rf194. 1: 100 bp marker, 2: homozygous insertion, 3: heterozygous insertion. (E) Sanger trace representing distal breakpoint of insertion. Distal breakpoint has been mapped to chromosome 1 between 109,650,634-109,650,635 (F) Schema representing the retrotransposition event.

to the exon/intron boundaries, suggestive for a retroposed BOD1 copy (Additional file 1: Figure S8). Furthermore informative SNPs located on the reads supporting the MEI event in $B O D 1$, suggest that the MEI event was located with the retroposed copy of $B O D 1$, and incorrectly anchored into $B O D 1$.

\section{Direct comparison of WES and WGS paired-end MEI calls in a CEU trio}

Next we investigated the accuracy of detecting MEI events in WES data (Additional file 1: Table S5), by determining the overlap between MEI predictions based on WGS data from the CEU trio (NA12878, NA12891, NA12892) and WES data from the same trio. Predicted MEI coordinates from the WGS data were intersected with the capture region list, revealing only one exonic MEI event in ZNF880. This event, present in the WGS data of all three individuals, was also detected in the WES data of all three individuals by Mobster. The same ZNF880 MEI event was also found and validated in our in-house experimental paired-end WES data (Table 4). Conversely all WES MEI events $(n=24)$ in this trio, including the predictions outside the official capture regions $(n=21)$, were also called in the WGS data. Demonstrating the reliability of Mobster in exome data, however more events are required in order to further investigate the influence of the capture step on detection sensitivity.

\section{Longer clipped reads have less chance of aligning at random to the mobilome}

Due to the short read length of $50 \mathrm{bp}$ for the single-end NGS data, we next investigated the required length for clipped sequences to reliably detect MEIs. Additional file 1: Table S1 summarizes how many reads out of 1,000,000 generated reads align against the mobilome using different mismatch settings in BWA. Based on these results we conclude that clipped reads of $20 \mathrm{bp}$ and 1 mismatch or higher could reliably map to the mobilome.

\section{Detecting MEls in single-end WES experimental data}

Finally 101 parent-child trios consisting of single-end whole exome sequencing were analyzed with Mobster. Predictions required support on both sides of the insertion, with at least five supporting reads that had an average clipped length of at least 20 bases, with the clipped bases having at least an average quality of 20 . In addition the clipping positions of reads on one side of the predicted MEI were not allowed to differ by more than 3 bp. Using this strategy, 89 putative exonic MEI events were found. In order to increase confidence, only predictions were considered that were present in more than one individual. Using this approach one MEI event remained, located in the second exon of C1orf194 and predicted in five individuals.

Validation of the predicted MEI event revealed a novel pseudogene created from L1 mediated retrotransposition of UQCR10 into C1orf194 (Figure 3D-F), resulting in a 
truncated C1orf194 protein. This retrotransposition event is estimated to occur in $15.5 \%$ of Caucasian individuals screened and present in both heterozygous and homozygous states.

\section{Discussion and conclusions}

We present a novel method (Mobster) able to reliably detect active non-reference MEI events in both pairedend and single-end WES and WGS sequence data. The estimated FDR of Mobster based upon validation experiments is $10 \%$ or less for paired-end WGS data across Alu and L1 events (Table 4). These results are supported by simulation data based on different read depths (see Additional file 1: Figures S1 and S2) and benchmarking with a public NGS dataset. Previous MEI detection methods show an increase in specificity with read depths but focus on WGS data with read lengths 100 bp or longer [17]. We show that it is possible to detect MEI events in WES as well as single-end short-read (50 bp) length data, and that reads 18 base pairs long with 0 mismatches can uniquely map to the mobilome. While reads clipped to 20 base pairs have a greater than 99.99\% chance of mapping uniquely when allowing for one mismatch and hence was used as the cutoff for our WES single-end analysis (Additional file 1: Table S1). This is in concordance with the predicted e-value which is calculated to be, respectively, $4.5 \times 10^{-4}$ and $4.1 \times 10^{-4}$.

While it is possible to detect MEI events in single-end short-read length data, both the sensitivity and specificity of Mobster improved on data with longer read lengths, and discordant paired-end information data. Many of the supporting reads for the MEI predictions were obtained by analyzing all discordant read pairs and not just those read pairs which are mapped multiple times. This demonstrates the importance of using all discordant read pairs in structural variation detection for increasing sensitivity. The 1,181 MEIs predicted in paired-end WGS data were supported on average by 47 (SD \pm 27 ) reads mapping to the mobilome or a homopolymer $(\mathrm{A} / \mathrm{T})$ stretch. The majority of these reads were originally mapped multiple times against the reference (64.6\%) or clipped (24.0\%). In contrast a small number of reads were originally mapped uniquely but discordant to the reference genome (8.8\%), or were unmapped (2.7\%). A minority of predicted MEIs were not supported by discordant read pairs on both sides of the event, but only by clipped reads $(n=47)$ (Additional file 1 : Figure S3). By allowing these predictions, Mobsters' sensitivity increases in genomic regions with lower coverage, hard to map regions and for heavily truncated insertion events. The majority of events detected in the single-end short read data were singletons with a higher rate of novel events, suggesting a higher false positive rate than with paired-end data. Validation of these events suggested a number of the split reads where the result of indels and not MEIs.

We determine that $4 \%$ to $5 \%$ of MEIs detected by Mobster are novel in comparison to a reference set of known MEI events consisting of dbRIP, events reported by Stewart et al. [17], Hormozdiari et al. [14], and Lee et al. [13]. This high overlap with previously reported MEIs suggests that Mobster has both a low false positive rate, which is supported by the FDR of the validation experiments, and that existing resources of pMEI events are currently incomplete. In comparison, low-coverage WGS in the pilot 1000 Genomes Project had a detection sensitivity of $70 \%$ to $80 \%$ for common (allele frequency $>0.1$ ) nonreference Alu insertion loci [17]. While high-coverage (approximately $15 \mathrm{X}$ to $40 \mathrm{X}$ ) sequencing with both long (Roche 454) and short (Illumina) reads was required to achieve a per-individual sensitivity of $90 \%$ [17].

Mobster detected on average 1,100 MEI events per individual using whole genome sequencing data of which approximately 436 were genic. Previous reports suggest that approximately 5,370 non-reference MEI exist in a dataset consisting of 179 samples, of which $42 \%$ are genic, and only a small number are exonic [17]. Both our results and those previously reported indicate a depletion of MEI events in the coding regions of the genome. No significant bias towards insertion into the first or last intron of a gene was observed. However an antisense orientation bias was observed for MEIs, arguing for a selection against sense MEIs. Sense MEIs terminate gene transcription more efficiently than anti-sense MEIs [32]. Mobile element insertions into the coding exons of genes are likely to disrupt gene function and therefore face strong purifying selection. Such insertions are expected to exist only briefly in the population, as very rare insertions, which require robust high throughput detection methods for identification. Capturing these rare MEI events will allow investigation into the factors influencing ME retrotransposition rates and site preferences, prior to potentially confounding influences such as natural selection, demographic changes, and post integration rearrangements.

The majority of MEI events are consistent in structure and their integration into the genome results often in either a target site duplication or sometimes a deletion [33]. Target site duplications were observed with the majority (93\%) of MEI events, while the remainder had either a target site deletion or no indel at the integration site. Deletions or duplications at the integration site occur after or during minus strand synthesis, in which a second strand nick of the target site occurs. Depending on the retrotransposon involved, the second strand nick can occur downstream, upstream, or in line with the bottom strand nick to generate target site duplications (TSDs), target site deletions, or blunt insertions [34,35]. We observed no statistically significant trend towards 
target site deletions or duplications dependent on the family of the MEI element (Alu, L1 or SVA). It has previously been observed that target site duplications and deletions tend to either be $15 \mathrm{nt}$ or 9 nt in length [33], more specifically we observe that the majority of target site duplications are $14 \mathrm{nt}$ in length, and target site deletions have a median of $7 \mathrm{nt}$ (Figure 2B).

In addition to MEI events, the processing of novel pseudogenes is also a form of retrotransposition. We detected such gene retrotransposition events in both the single-end and paired-end WES datasets. The first and validated event involved an exonic integration site involving an L1 element, whereby UQCR10 is inserted into exon 2 of C1orf194. (Figure 3D to F). This event was recently detected in silico [36]. Based on the analysis of 101 trios we estimate that this non-reference event has a minor allele frequency of 0.15 in the Caucasian population. An additional L1 retrotransposed gene was detected in the WES paired-end dataset involving BOD1 in both the child and parental DNA. The resulting non-reference pseudogene shows evidence of exons 2 to 4 being fused (Additional file 1: Figure S8). Numerous retrotransposed versions of $B O D 1$ have previously been reported, the closest matching known element, BOD1L2, has more than 26 mismatches as well as a 6 bp gap leading to the conclusion that the event observed is novel and that BOD1 could be considered a hotspot for such events. Similar to MEI events the retrotransposition of novel pseudogenes results in structural variation which may lead to disease or result in normal genomic variation.

The development of robust algorithms to detect MEI events in NGS data is important for calculating an accurate de novo insertion rate for mobile elements. Previous de novo rates have been estimated indirectly using phylogenetic and population methods [37-39]. The relative retrotransposition rates for the three element classes $A l u, \mathrm{~L} 1$, and SVA are estimated to be $0.039,0.0056$, and 0.002 insertions per genome per generation, respectively. However, phylogenetic and population methods will not detect MEIs that are lost soon after integration. The de novo insertion rate can be directly obtained using trio data. We present a method for the detection of MEI events in a variety of NGS data and explore some of the genomic properties of these events. The application of this method to larger cohorts would detect additional novel MEIs with potentially important functional consequences as well as retrotransposed gene events.

\section{Additional files}

Additional file 1: Supplementary results, figures and tables. Additional file 2: Mobster's double cluster calls on NA12878. Additional file 3: Mobster's double cluster calls on NA12891. Additional file 4: Mobster's double cluster calls on NA12892.
Competing interests

The authors declare that they have no competing interests.

\section{Authors' contributions}

DTT and JdL developed Mobster. DTT, JdL, and JHK wrote the manuscript. $J H K$ and JV designed the study. LV, MS, and PdV performed validation experiments. MK, KY, and ES provided samples and software for comparison and validation. All authors read and approved the final manuscript.

\section{Acknowledgements}

DT Thung, M Steehouwer, and JA Veltman are supported by the European Research Council (DENOVO 281964 to JA Veltman). We also wish to thank A. Hoischen for providing access to the paired-end WES dataset and validation samples. This publication was supported by the Dutch national program COMMIT.

\section{Author details}

${ }^{1}$ Department of Human Genetics, RadboudUMC, P.O. Box 9101, 6500, HB, Nijmegen, the Netherlands. 'Department of Molecular Epidemiology, Leiden University Medical Centre, Leiden, The Netherlands. ${ }^{3}$ The Genome Institute, Washington University, St Louis, Missouri, USA. ${ }^{4}$ Hubrecht Institute, KNAW, Utrecht, The Netherlands. ${ }^{5}$ Department of Clinical Genetics, Maastricht University Medical Centre, Maastricht, The Netherlands.

Received: 6 January 2014 Accepted: 7 October 2014

Published online: 28 October 2014

\section{References}

1. Beck CR, Garcia-Perez JL, Badge RM, Moran JV: LINE-1 elements in structural variation and disease. Annu Rev Genomics Hum Genet 2011, 12:187-215.

2. Cordaux R, Batzer MA: The impact of retrotransposons on human genome evolution. Nat Rev Genet 2009, 10:691-703.

3. Lander ES, Linton LM, Birren B, Nusbaum C, Zody MC, Baldwin J, Devon K, Dewar K, Doyle M, FitzHugh W, Funke R, Gage D, Harris K, Heaford A, Howland J, Kann L, Lehoczky J, LeVine R, McEwan P, McKernan K, Meldrim J, Mesirov JP, Miranda C, Morris W, Naylor J, Raymond C, Rosetti M, Santos R, Sheridan A, Sougnez C, et al: Initial sequencing and analysis of the human genome. Nature 2001, 409:860-921.

4. de Koning APJ, Gu W, Castoe TA, Batzer MA, Pollock DD: Repetitive elements may comprise over two-thirds of the human genome. PLOS Genet 2011, 7:e1002384.

5. Mills RE, Bennett EA, Iskow RC, Devine SE: Which transposable elements are active in the human genome? Trends Genet 2007, 23:183-191.

6. Brouha B, Schustak J, Badge RM, Lutz-Prigge S, Farley AH, Moran JV, Kazazian HH: Hot L1s account for the bulk of retrotransposition in the human population. Proc Natl Acad Sci 2003, 100:5280-5285.

7. Bennett EA, Keller H, Mills RE, Schmidt S, Moran JV, Weichenrieder O, Devine SE: Active Alu retrotransposons in the human genome. Genome Res 2008, 18:1875-1883.

8. Hancks DC, Kazazian HH Jr: Active human retrotransposons: variation and disease. Curr Opin Genet Dev 2012, 22:191-203.

9. Sen SK, Han K, Wang J, Lee J, Wang H, Callinan PA, Dyer M, Cordaux R, Liang $P$, Batzer MA: Human genomic deletions mediated by recombination between Alu elements. Am J Hum Genet 2006, 79:41-53.

10. Kazazian HH, Wong C, Youssoufian H, Scott AF, Phillips DG, Antonarakis SE: Haemophilia a resulting from de novo insertion of $L 1$ sequences represents a novel mechanism for mutation in man. Nature 1988, 332:164-166.

11. Iskow RC, McCabe MT, Mills RE, Torene S, Pittard WS, Neuwald AF, Van Meir EG, Vertino PM, Devine SE: Natural mutagenesis of human genomes by endogenous retrotransposons. Cell 2010, 141:1253-1261.

12. Solyom S, Ewing AD, Rahrmann EP, Doucet T, Nelson HH, Burns MB, Harris RS, Sigmon DF, Casella A, Erlanger B, Wheelan S, Upton KR, Shukla R, Faulkner GJ, Largaespada DA, Kazazian HH Jr: Extensive somatic L1 retrotransposition in colorectal tumors. Genome Res 2012, 22:2328-2338.

13. Lee $E$, Iskow R, Yang L, Gokcumen O, Haseley P, Luquette LJ, Lohr JG, Harris CC, Ding L, Wilson RK, Wheeler DA, Gibbs RA, Kucherlapati R, Lee C, Kharchenko PV, Park PJ: Landscape of somatic retrotransposition in human cancers. Science 2012, 337:967-971.

14. Hormozdiari F, Alkan C, Ventura M, Hajirasouliha I, Malig M, Hach F, Yorukoglu D, Dao P, Bakhshi M, Sahinalp SC, Eichler EE: Alu repeat 
discovery and characterization within human genomes. Genome Res 2011, 21:840-849.

15. Ewing $\mathrm{AD}$, Kazazian $\mathrm{HH}$ : Whole-genome resequencing allows detection of many rare LINE-1 insertion alleles in humans. Genome Res 2011, 21:985-990.

16. Keane TM, Wong K, Adams DJ: RetroSeq: transposable element discovery from next-generation sequencing data. Bioinformatics 2013, 29:389-390.

17. Stewart C, Kural D, Strömberg MP, Walker JA, Konkel MK, Stütz AM, Urban AE, Grubert F, Lam HYK, Lee W-P, Busby M, Indap AR, Garrison E, Huff C, Xing J, Snyder MP, Jorde LB, Batzer MA, Korbel JO, Marth GT, 1000 Genomes Project: A comprehensive map of mobile element insertion polymorphisms in humans. PLOS Genet 2011, 7:e1002236.

18. Picard. [http://broadinstitute.github.io/picard/]

19. Jurka J: Repbase update: a database and an electronic journal of repetitive elements. Trends Genet 2000, 16:418-420.

20. Wang K, Li M, Hakonarson H: ANNOVAR: functional annotation of genetic variants from high-throughput sequencing data. Nucleic Acids Res 2010, 38:e164.

21. Quinlan AR, Hall IM: BEDTools: a flexible suite of utilities for comparing genomic features. Bioinformatics 2010, 26:841-842.

22. Crooks GE, Hon G, Chandonia JM, Brenner SE: WebLogo: a sequence logo generator. Genome Res 2004, 14:1188-1190.

23. Tangram: Wu J, Lee WP, Ward A, Walker JA, Konkel MK, Batzer MA, Marth GT: Tangram: a comprehensive toolbox for mobile element insertion detection. BMC Genomics 2014, 15:795.

24. David M, Mustafa $H$, Brudno M: Detecting Alu insertions from highthroughput sequencing data. Nucleic Acids Res 2013, 41:e169.

25. CEU Trio high coverage WEx and WGS dataset. [ftp://ftp-trace.ncbi.nih. gov/1000genomes/ftp/technical/working/20120117_ceu_trio_b37_decoy/]

26. CEU Trio MEI calls of RetroSeq, Tangram and TEA. [ftp://ftp-mouse.sanger. ac.uk/other/tk2/RetroSeq/CEU_trio/]

27. Exome capture intervals for WEx data of CEU Trio. [ftp://gsapubftpanonymous@ftp.broadinstitute.org/bundle/2.8/b37/Broad.human.exome.b37. interval_list.gz]

28. Li H, Durbin R: Fast and accurate short read alignment with BurrowsWheeler transform. Bioinformatics 2009, 25:1754-1760.

29. Lee W-P, Stromberg MP, Ward A, Stewart C, Garrison EP, Marth GT: MOSAIK: a hash-based algorithm for accurate next-generation sequencing short-read mapping. PLoS One 2014, 9:e90581.

30. de Ligt J, Willemsen MH, van Bon BW, Kleefstra T, Yntema HG, Kroes T, Vulto-van Silfhout AT, Koolen DA, de Vries P, Gilissen C, del Rosario M Hoischen A, Scheffer $H$, de Vries BB, Brunner HG, Veltman JA, Vissers LE: Diagnostic exome sequencing in persons with severe intellectual disability. N Engl J Med 2012, 367:1921-1929.

31. Cost GJ, Boeke JD: Targeting of human retrotransposon integration is directed by the specificity of the $L 1$ endonuclease for regions of unusual DNA structuret. Biochemistry 1998, 37:18081-18093.

32. Conley $A B$, Jordan IK: Cell type-specific termination of transcription by transposable element sequences. Mob DNA 2012, 3:15.

33. Szak ST, Pickeral OK, Makalowski W, Boguski MS, Landsman D, Boeke JD: Molecular archeology of L1 insertions in the human genome. Genome Biol 2002, 3:research0052.

34. Symer DE, Connelly C, Szak ST, Caputo EM, Cost GJ, Parmigiani G, Boeke JD: Human I1 retrotransposition is associated with genetic instability in vivo. Cell 2002, 110:327-338.

35. Zingler N, Willhoeft U, Brose HP, Schoder V, Jahns T, Hanschmann KM, Morrish TA, Lower J, Schumann GG: Analysis of $5^{\prime}$ junctions of human LINE-1 and Alu retrotransposons suggests an alternative model for 5 '-end attachment requiring microhomology-mediated end-joining. Genome Res 2005, 15:780-789.

36. Ewing A, Ballinger T, Earl D, Sequencing BIG, Program A, Platform, Harris C, Ding L, Wilson R, Haussler D: Retrotransposition of gene transcripts leads to structural variation in mammalian genomes. Genome Biol 2013, 14:R22.

37. Cordaux R, Hedges DJ, Herke SW, Batzer MA: Estimating the retrotransposition rate of human Alu elements. Gene 2006, 373:134-137.
38. Ewing $A D$, Kazazian $H H$ : High-throughput sequencing reveals extensive variation in human-specific L1 content in individual human genomes. Genome Res 2010, 20:1262-1270.

39. Xing J, Zhang Y, Han K, Salem AH, Sen SK, Huff CD, Zhou Q, Kirkness EF, Levy S, Batzer MA, Jorde LB: Mobile elements create structural variation: analysis of a complete human genome. Genome Res 2009, 19:1516-1526.

doi:10.1186/s13059-014-0488-

Cite this article as: Thung et al.: Mobster: accurate detection of mobile element insertions in next generation sequencing data. Genome Biology 2014 15:488

\section{Submit your next manuscript to BioMed Central and take full advantage of:}

- Convenient online submission

- Thorough peer review

- No space constraints or color figure charges

- Immediate publication on acceptance

- Inclusion in PubMed, CAS, Scopus and Google Scholar

- Research which is freely available for redistribution

Submit your manuscript at www.biomedcentral.com/submit
C Biomed Central 